\title{
Avaliação nutricional de infantes: Diagnóstico e propedêutica empírica nas escolas
}

\author{
Nutritional assessment of infants: Diagnosis and empirical workup in schools \\ Evaluación nutricional de los lactantes: Diagnóstico y evaluación empírica en las escuelas
}

Recebido: 17/07/2021 | Revisado: 21/07/2021 | Aceito: 22/07/2021 | Publicado: 30/07/2021

Caio Flávio Reis Nogueira

ORCID: https://orcid.org/0000-0003-1514-9660

Centro Universitário de Patos de Minas, Brasil caioflavio@unipam.edu.br

Bárbara Queiroz de Figueiredo ORCID: https://orcid.org/0000-0003-1630-4597 Centro Universitário de Patos de Minas, Brasil barbarafigueiredo@unipam.edu.br

Letícia Beatriz Rodrigues Bernardes ORCID: https://orcid.org/0000-0001-5760-214X Centro Universitário de Patos de Minas, Brasil leticiabernardes@unipam.edu.br

Rúbia Carla Oliveira

ORCID: https://orcid.org/0000-0002-7162-3497 Centro Universitário de Patos de Minas, Brasil rubiacoliveira@unipam.edu.br

\begin{abstract}
Resumo
Introdução: O estado nutricional é dado como "o estado resultante do equilíbrio entre o suprimento de nutrientes e o gasto energético". As alterações do estado nutricional (desnutrição e obesidade) são relacionadas com sérios agravos para a saúde. Destaca-se a importante influência do monitoramento do estado nutricional na fase da infância no processo de crescimento e desenvolvimento. Objetivo: descrever os principais métodos de avaliação do estado nutricional em crianças, com finalidade de facilitar o diagnóstico dos problemas nutricionais e acompanhar intervenções dietoterápicas. Metodologia: Trata-se de uma pesquisa descritiva do tipo breve revisão integrativa da literatura, que buscou conceituar estado nutricional e métodos de avaliação nutricional em infantes. Como critérios de inclusão, foram considerados artigos originais, que abordassem o tema pesquisado e permitissem acesso integral ao conteúdo do estudo, publicados no período de 1999 a 2021, em inglês, espanhol e português. Resultados: Descreve o método clínico, detalha aspectos da antropometria e os exames laboratoriais utilizados em avaliação do estado nutricional. Relaciona alguns dos principais índices múltiplos, utilizados tanto com fins diagnósticos e prognósticos. Além disso, é capaz de rastrear, por meio de entrevistas, possíveis repetições familiares relacionadas a desequilíbrios hormonais e síndromes metabólicas. Conclusão: Diante disso, faz-se necessário alertar a população dos riscos nutricionais que podem surgir durante a infância, ressaltando a importância da reeducação alimentar e atividades de educação nutricional nas escolas, as quais podem auxiliar na formação de hábitos alimentares saudáveis, na manutenção da saúde e controle de peso corporal adequado.
\end{abstract}

Palavras-chave: Antropometria; Nutrição; Crianças; Projeto Saúde na Escola; Educação.

\begin{abstract}
Introduction: Nutritional status is given as "the state resulting from the balance between nutrient supply and energy expenditure". Changes in nutritional status (malnutrition and obesity) are related to serious health problems. The important influence of monitoring nutritional status during childhood on the growth and development process is highlighted. Objective: to describe the main methods of assessment of nutritional status in children, in order to facilitate the diagnosis of nutritional problems and monitor dietary interventions. Methodology: This is a descriptive research of a brief integrative literature review, which sought to conceptualize nutritional status and nutritional assessment methods in infants. As inclusion criteria, original articles that addressed the research topic and allowed full access to the study content, published from 1999 to 2021, in English, Spanish and Portuguese, were considered. Results: Describes the clinical method, details aspects of anthropometry and laboratory tests used to assess nutritional status. Lists some of the main multiple indices used for both diagnostic and prognostic purposes. In addition, it is able to track, through interviews, possible family repetitions related to hormonal imbalances and metabolic syndromes. Conclusion: Therefore, it is necessary to alert the population to the nutritional risks that may arise during childhood, emphasizing the importance of food re-education and nutrition education activities in schools, which can help in the formation of healthy eating habits, in the maintenance of health and proper body weight control.
\end{abstract}

Keywords: Anthropometry; Nutrition; Kids; Health at School Project; Education. 


\begin{abstract}
Resumen
Introducción: El estado nutricional se da como "el estado resultante del equilibrio entre el aporte de nutrientes y el gasto energético". Los cambios en el estado nutricional (desnutrición y obesidad) están relacionados con graves problemas de salud. Se destaca la importante influencia del seguimiento del estado nutricional durante la infancia en el proceso de crecimiento y desarrollo. Objetivo: describir los principales métodos de evaluación del estado nutricional en ninos, con el fin de facilitar el diagnóstico de problemas nutricionales y monitorear las intervenciones dietéticas. Metodología: Se trata de una investigación descriptiva de una breve revisión integradora de la literatura, que buscó conceptualizar el estado nutricional y los métodos de evaluación nutricional en lactantes. Como criterios de inclusión, se consideraron artículos originales que abordaran el tema investigado y permitieran el pleno acceso al contenido del estudio, publicados de 1999 a 2021, en inglés, español y portugués. Resultados: Describe el método clínico, detalla aspectos de antropometría y pruebas de laboratorio utilizadas para evaluar el estado nutricional. Enumera algunos de los principales índices múltiples que se utilizan tanto con fines de diagnóstico como de pronóstico. Además, es capaz de rastrear, a través de entrevistas, posibles repeticiones familiares relacionadas con desequilibrios hormonales y síndromes metabólicos. Conclusión: Por lo tanto, es necesario alertar a la población sobre los riesgos nutricionales que pueden surgir durante la infancia, enfatizando la importancia de las actividades de reeducación alimentaria y educación nutricional en las escuelas, que pueden ayudar en la formación de hábitos alimentarios saludables, en de salud y control adecuado del peso corporal.
\end{abstract}

Palabras clave: Antropometría; Nutrición; Niños; Proyecto Salud en la Escuela; Educación.

\title{
1. Introdução
}

Sabe-se que a Unidade Básica de Saúde (UBS) é a porta de entrada do Sistema Único de Saúde (SUS) e está relacionada com nível de atenção primário à saúde cujo grau de complexidade dos atendimentos é menor bem como o grau das tecnologias utilizadas. Em contrapartida, é indiscutível a importância dos serviços prestados pela UBS, sendo extremamente pertinentes nos aspectos saúde-doença dos cidadãos brasileiros. Desse modo, aliado a uma equipe multiprofissional, os profissionais que compõem esse modelo de unidade de saúde atuam de forma evidentemente eficaz, com uma resolubilidade de cerca de 80\% dos casos por meio das ações de prevenção e promoção à saúde (Brasil, 2018).

Um exemplo da relevância das UBS em consonância com as equipes da Estratégia Saúde da Família (ESF) é o projeto Saúde na Escola, o qual articula educação e saúde em um mesmo ambiente. Esse projeto é de suma importância, pois, por meio dessa relação mais estreita que as ESF conseguem estabelecer com a comunidade é possível realizar o monitoramento e a avaliação da saúde dos estudantes no âmbito escolar e fora dele, analisando a vulnerabilidade as quais eles possam estar expostos. Assim, torna-se possível e viável o desenvolvimento de estratégias que possibilitem a prevenção e promoção da saúde para esses estudantes (Starfield, 2002).

A alimentação adequada constitui um direito social fundamental, entretanto, a realidade observada no Brasil demonstra que existem problemas quanto a esse respeito. Diante disso, a avaliação do estado nutricional relaciona-se como um instrumento de diagnóstico social e clínico sendo considerada um ótimo indicador de qualidade de vida. Esse aspecto corrobora a importância da abordagem nutricional em todas as fases da vida, principalmente na infância e adolescência, porque, além de revelar as possíveis condições às quais essas pessoas possam estar expostas, seja de miséria ou de desinformação, poderá indicar também, propensão ao desenvolvimento de determinadas doenças (Mello, 2002),

Ainda que exista o projeto Saúde na escola, é evidente que a saúde das crianças e adolescentes necessitam de um acompanhamento ainda mais rigoroso, devido à fase em que se encontram, de desenvolvimento físico e intelectual, e por estarem mais suscetíveis a influências diversas. Diante disso, a nutrição dos indivíduos representa um aspecto significativo a ser abordado, tendo em vista as correlações existentes entre a alimentação e os aspectos biológicos, como o crescimento e desenvolvimento do ser humano, além das relações clínicas, como a obesidade e desnutrição, e também pelo fato de apresentar-se como fator de risco para algumas patologias como a hipertensão e diabetes. Com isso, no ano de 2011 o Ministério da Saúde desenvolveu o Plano Nacional de Enfrentamento das Doenças Crônicas não Transmissíveis (DNCT), que estão bastante associadas à problemas no aparelho circulatório e também ao diabetes mellitus, sendo a principal causa de morte prematura no Brasil. Esse Plano tem como objetivo priorizar maneiras de prevenir essas DCNT e sabe -se que a obesidade é 
um fator de risco para essas doenças, sendo esse mais um fator que explicita a necessidade e políticas voltadas para a avaliação do estado nutricional da população (Fonseca, 2013).

Por conseguinte, a prevenção deve ser a primeira linha de defesa contra as complicações decorridas de um estado nutricional inadequado. Dessa forma, a existência de programas educacionais abrangentes, que incluem o acompanhamento dos estudantes, classificação de risco e educação informativa, podem melhorar os estados nutricionais que iremos observar nesse ambiente, seja ele voltado para a obesidade ou desnutrição. Os alunos, ao terem acesso às informações necessárias sobre as vertentes de uma alimentação adequada poderão estabelecer melhorias no seu estado nutricional, bem como influenciar toda a rede em que eles convivem (Starfield, 2002).

A vigilância e a monitorização do crescimento constituem ações fundamentais no atendimento à criança. $\mathrm{O}$ crescimento é um processo bastante sensível às diversidades do meio onde a criança cresce e deve ser utilizado como indicador da saúde infantil. A avaliação do crescimento é a medida que melhor define a saúde e o estado nutricional de crianças, já que distúrbios na saúde e nutrição, independentemente de suas etiologias, afetam o crescimento infantil. A maioria dos problemas de saúde e nutrição durante a infância, na opinião dos mesmos autores, está relacionada ao consumo alimentar inadequado e às infecções de repetição. Essas duas condições são descritas como resultado do padrão de vida da população, que inclui dificuldades sociais e econômicas que se refletem em condições inadequadas de moradia, comprometem a aquisição de uma alimentação balanceada e dificultam o acesso aos serviços de saúde. Ademais, os distúrbios nutricionais da infância têm consequências sérias que tornam imperativos a sua prevenção e o seu controle.

Outrossim, a obesidade é uma das principais moléstias da atualidade, haja vista suas altas taxas de prevalência e incidência. Constitui um fator de risco importante que desencadeia doenças crônicas e complicações na saúde humana, sejam elas cardiovasculares, endócrino-metabólicas e psicossociais, sendo um catalizador de destaque para enredos secundários como infertilidade, imunodepressão e frustrações transcendentes. É notório que a obesidade infantil vem aumentando exponencialmente, e que determina várias complicações na infância e, por conseguinte, na fase adulta.

A etiologia da obesidade ilustra uma problemática acumulativa multifatorial que envolve aspectos ambientais, genéticos e habituais. Atualmente, é um problema de saúde pública mundial. Sabe-se, também, que a transição nutricional caracteriza um processo de modificações sequenciais nos padrões de nutrição e consumo, que acompanha mudanças econômicas, sociais, demográficas e culturais. O presente estudo tem como objetivo conceituar estado nutricional e métodos de avaliação nutricional em infantes, pois por ser uma doença muitas das vezes, reversível, a valia do trabalho profilático realizado com o espaço amostral infantil e adolescente, é qualitativo e empírico.

\section{Metodologia}

Trata-se de uma pesquisa descritiva do tipo breve revisão integrativa da literatura, que buscou conceituar estado nutricional e métodos de avaliação nutricional em infantes. A pesquisa foi realizada através do acesso online nas bases de dados National Library of Medicine (PubMed MEDLINE), Scientific Electronic Library Online (Scielo), Cochrane Database of Systematic Reviews (CDSR), Google Scholar, Biblioteca Virtual em Saúde (BVS) e EBSCO Information Services, nos meses de abril a junho de 2021.

Para a busca das obras foram utilizadas as palavras-chaves presentes nos descritores em Ciências da Saúde (DeCS): em inglês: "Anthropometry", "Nutrition", "Children", "Health at School Project", "Education", em português: "Antropometria", "Nutrição", "Crianças", "Projeto Saúde na Escola", "Educação" e em espanhol: "Antropometría", "Nutrición", "Niños", "Proyecto Salud en la Escuela", "Educación". Como critérios de inclusão, foram considerados artigos originais, que abordassem o tema pesquisado e permitissem acesso integral ao conteúdo do estudo, publicados no período de 1999 a 2021, em inglês, espanhol e português. O critério de exclusão foi imposto naqueles trabalhos que não estavam em 
inglês ou português, que não tinham passado por processo de Peer-View e que não relacionassem a temática de avaliação nutricional em infantes.

A estratégia de seleção dos artigos seguiu as seguintes etapas: busca nas bases de dados selecionadas; leitura dos títulos de todos os artigos encontrados e exclusão daqueles que não abordavam o assunto; leitura crítica dos resumos dos artigos e leitura na íntegra dos artigos selecionados nas etapas anteriores. Após leitura criteriosa das publicações, 6 artigos não foram utilizados devido aos critérios de exclusão. Assim, totalizaram-se 19 artigos científicos para a revisão integrativa da literatura, com os descritores apresentados acima. Após esta seleção, filtraram-se por artigos dos últimos vinte e dois anos e por artigos em línguas portuguesa, espanhola e inglesa.

\section{Resultados e Discussão}

Os padrões e hábitos alimentares da população vêm mudando nos últimos anos, principalmente em virtude das transformações que marcam o mundo contemporâneo, tais como a urbanização, a industrialização crescente, a relação entre tempo e espaço, as múltiplas atribuições da mulher na família e no trabalho, entre outras (Henriques, et al., 2012). Estes fatores reforçam a substituição de alimentos in natura ou minimamente processados, por alimentos ultraprocessados e/ou refeições fora de casa, devido à sua praticidade e economia de tempo. Com isso, as crianças são expostas cada vez mais cedo a uma alimentação desequilibrada, pobre em nutrientes e excessiva em calorias (Brasil, 2014).

Além disso, na era do imediatismo, da correria e da falta de tempo dos pais, a televisão, o computador, o tablet e o celular tornaram-se os companheiros prediletos das crianças, exercendo papel socializador e influenciando, entre outras coisas, nas práticas alimentares. Enquanto os pais trabalham o dia todo e chegam em casa exaustos, as crianças ficam expostas ao que a mídia fala, exibe e ensina (Lima, 2010). Paralelo a isso, há um crescimento constante na promoção de alimentos industrializados, o que tem influenciado negativamente a alimentação e a saúde das crianças (Moura, 2010). Além do mais, a divulgação de alimentos ultraprocessados domina os anúncios comerciais, propagando muitas vezes informações incompletas ou incorretas sobre alimentação, e atingindo principalmente as crianças (Brasil, 2014).

As mudanças nos padrões alimentares das crianças seguem uma tendência global na direção de uma alimentação cada vez mais rica em fontes de carboidratos e calorias (Rodrigues, et al., 2012). O elevado consumo de alimentos ultraprocessados e do tipo fast food (seja por influência da mídia, da família ou amigos) faz com que as crianças comecem cada vez mais cedo um hábito alimentar incorreto, que pode acarretar, entre outros problemas, na obesidade infantil (Porto, et al., 2013). A indústria alimentar vê a criança como sujeito consumidor e está cada vez mais atenta ao fato de elas terem grande influência na decisão de compra dos pais (Rodrigues, et al., 2011), devido ao seu considerável poder de importunação (Henriques, et al., 2012).

Os problemas nutricionais são, indubitavelmente, um dos mais importantes problemas de saúde pública mundialmente, estão diretamente ligados às condições socioeconômicas e culturais menos favorecidas e têm como sua maior vítima, a criança. Neste contexto, cabe ressaltar que uma das maneiras de prevenir, diagnosticar e tratar os casos de morbidades infantis se caracteriza por ações que visem à melhora e controle de possíveis distúrbios alimentares. Uma dessas ações referese à avaliação nutricional (Freitas, et al., 2018).

\subsection{Conceito de estado nutricional}

A nutrição adequada é essencial para o bem-estar de qualquer sociedade ou indivíduo. A pouca variedade, os desequilíbrios na qualidade e na quantidade de alimentos disponíveis, bem como o padrão irregular de ingestão alimentar, podem afetar profundamente a saúde. O estado nutricional reflete o grau no qual as necessidades fisiológicas de nutrientes estão sendo ou não atendidas. O equilíbrio ente a ingestão de nutrientes e as necessidades do organismo determinam um 
estado nutricional ótimo. A ingestão de nutrientes depende do consumo real de alimentos e é influenciada por fatores socioeconômicos, culturais, comportamento alimentar, ambiente emocional e patologias (que podem afetar a capacidade de comer e absorver nutrientes). As necessidades de nutrientes irão depender de fatores como: estresse, infecções, trauma, crescimento, gravidez, manutenção do peso, estresse psicológico (Brasil, 2018)

As informações sobre as ingestões reais são obtidas por meio da observação direta ou de um inventário de alimentos consumidos. São vários os métodos de investigação alimentar, selecionamos dois métodos que podem ser utilizados mais facilmente: são aquele em que o entrevistador registra tudo o que o indivíduo consome em 24 horas através de um recordatório (R24); e aquele em que o indivíduo registra tudo o que come num período de 3 a 7 dias (Registro Alimentar). $\mathrm{O}$ entrevistador ou o indivíduo a ser analisado deve ser treinado para fazer esse tipo de levantamento de dados. A ingestão relacionada no relatório é analisada e a quantidade ingerida de cada nutriente é comparada com as ingestões dietéticas de referência (DRI) ou com as diretrizes do guia da Pirâmide Alimentar. Deve-se observar, além dos alimentos ingeridos, as quantidades de cada um em medidas caseiras (uma colher de sopa cheia ou rasa, copo de requeijão ou americano, escumadeira ou concha) ou em gramas. O local e horário das refeições são necessários para poder fazer correções como não comer assistindo TV, comer sentado, devagar, não consumir fast-food todos os dias (Krause, 2005).

Dessa maneira, o monitoramento do estado nutricional é importante para todas as faixas etárias, voltadas para a fase da infância e da adolescência, pois sua importância, nestes períodos de vida, advém do acompanhamento do processo de crescimento e desenvolvimento. Para o acompanhamento do estado nutricional, existem as medidas antropométricas que são utilizadas desde o século XVIII como instrumento de avaliação da saúde, porém, somente há menos de 40 anos, foram normatizadas para avaliação do estado nutricional individual e de populações. Nesse sentido, as medidas de peso e estatura são consideradas de alta sensibilidade, particularmente durante a idade pré-escolar, para refletir variações nas condições nutricionais. Dessa forma, os índices antropométricos podem ser tomados como indicadores positivos de saúde, pois permitem avaliar o estado nutricional e o potencial de desenvolvimento (Sampaio, 2012).

\subsection{Nutrição em idade escolar}

O período da educação infantil engloba crianças entre 0 a 6 anos de idade, sendo que o crescimento neste período é lento, mas constante, ocorrendo crescente maturação das habilidades motoras e ganho no crescimento cognitivo, social e emocional. $\mathrm{O}$ trato gastrointestinal dos escolares já atingiu a capacidade digestiva semelhante à do adulto, por isso possuem condições de receber a alimentação própria da família. Além disso, a creche e a pré-escola, constituem um ambiente e nicho que apresentam elevada vulnerabilidade biológica, sujeita a diversos agravos nutricionais, além de situações de falta de apetite às refeições básicas e alimentos específicos. Isto decorre de vários fatores que podem estar relacionados a uma maior seletividade em relação aos alimentos, fácil acesso a guloseimas, além da incidência de infecções e verminoses que podem diminuir o apetite. Esta fase é caracterizada pelo amadurecimento da habilidade motora, da linguagem e das habilidades sociais relacionadas à alimentação, sendo este um grupo vulnerável que depende dos pais ou responsáveis para receber alimentação adequada. A fase pré-escolar envolve comportamentos e atitudes que persistirão no futuro, podendo determinar uma vida saudável, a medida que um conjunto de ações que envolvem o ambiente familiar e escolar forem favoráveis ao estímulo e a garantia de práticas alimentares adequadas (Vasconcelos, 2011).

A fase escolar compreende crianças de 7 anos a 10 anos incompletos e é caracterizada por um período de crescimento e demandas nutricionais elevadas. $\mathrm{O}$ cardápio das crianças nessa faixa etária já está adaptado às disponibilidades e costumes dietéticos da família. Assim, é importante, reforçar às famílias sobre a importância de uma alimentação saudável e equilibrada, pois isso irá refletir na saúde da criança da mesma forma. Nessa fase, é comum a criança ter um alto gasto energético devido ao metabolismo que é mais intenso que o do adulto. Além disso, há nessa faixa etária intensa atividade 
física e mental. Assim, a falta de apetite comum à fase pré-escolar é substituída por um apetite voraz. É comum, nessa idade, também, a diminuição da ingestão de leite e, consequentemente, limitação do suprimento de cálcio. As mães devem estar atentas a fim de compensar a falta de ingestão de leite por meio de outros alimentos ricos em cálcio. Neste período as crianças apresentam necessidades nutricionais mais elevadas, bem como maior interesse pelos alimentos. É notório que os conceitos sobre nutrição são abstratos para os escolares, por isso devem ser fixados utilizando-se experiências significantes para que se tenham resultados positivos. Atividades que envolvam o preparo de alimentos, manejo de hortas e pomares, bem como oficinas culinárias e visitas a produtores agrícolas promovem às crianças uma ótima oportunidade de praticar e fortalecer seu conhecimento nutricional (Brasil, 2005).

Neste sentido, verifica-sea importância de programas de alimentação escolar que promovam a incorporação e manutenção de hábitos alimentares saudáveis, de forma a contribuir para a prevenção de carências nutricionais, bem como do excesso de peso. É importante considerar que as práticas alimentares são adquiridas durante toda a vida, destacando-se os primeiros anos como um período muito importante para o estabelecimento de hábitos alimentares que promovam a saúde do indivíduo desde a infância até a idade adulta. A escola, por sua vez, exerce notável influência na formação de crianças e adolescentes constituindo-se num centro de convivência e ensino-aprendizagem, aonde deve haver um envolvimento de toda a comunidade escolar, alunos, professores, funcionários, pais e nutricionista, que participem de forma integrada em estratégias e programas de promoção da alimentação saudável, garantindo assim a qualidade das refeições servidas (Brasil, 2005).

\subsection{Semiologia nutricional}

No campo das habilidades clínicas, a semiologia consiste em uma das ferramentas fundamentais para a avaliação nutricional, haja vista que por meio dela é possível extrair dados e compará-los a padrões de normalidade. Nesse sentido, algumas alterações no âmbito da nutrição de escolares podem ser identificadas a partir de tal ferramenta como a desnutrição, sobrepeso ou obesidade, por exemplo. Para tal intento, isto é, avaliação do estado nutritivo, a semiologia nutricional se vale de alguns métodos sendo eles a anamnese, exame físico, antropometria e exames laboratoriais (Sampaio, 2012).

A anamnese consiste em uma recordação de informações ou eventos importantes atrelados à saúde, bem como pode ser tida igualmente como um momento em que é possível identificar sintomas e sinais. No âmbito nutricional a anamnese possibilita aproximação entre o examinador e o paciente, bem como permite que o examinador compreenda a realidade do paciente e, a partir disso, trace diagnósticos, tratamentos e até medidas preventivas capazes de reduzir doenças atreladas a alterações nutricionais Para que a anamnese de escolares ocorra ela deve ser norteada por perguntas que permitam a identificação (nome, idade, sexo, endereço, naturalidade, procedência, escolaridade, filiação), bem como análises de hábitos de vida (pratica algum exercício físico, se sim, qual modalidade e com qual frequência, ou ainda se é sedentário), hábitos alimentares (quantas refeições são feitas por dia e em quais horários e locais, se há uso de suplementos ou polivitamínicos e tipos de alimentos mais ingeridos), desordens funcionais (apresenta náuseas, vômitos, diarreias, anorexia, pirose, disfagia, odinofagia, obstipação), assim como o histórico familiar (algum familiar apresenta alguma alteração nutricional) (Sampaio, 2012).

Consistindo no segundo método da avaliação clínica, o exame físico propicia uma avaliação física do paciente, obedecendo uma ordem (da cabeça aos pés) e valendo-se de quatro técnicas fundamentais, quais sejam, a inspeção, palpação, percussão e a ausculta. Em relação ao estado nutricional, o exame físico permite a obtenção e avaliação de dados importantes como a massa muscular, a gordura subcutânea o abdômen, bem como a presença de edemas e de alterações de coloração nas mucosas, pele e também na conjuntiva. Algumas alterações nutricionais como a desnutrição, a obesidade, bócio (carência de 
iodo) e xerose (carência de vitamina A), por exemplo, podem ser inferidas a partir dos dados coletados em tal exame. (Mussoi, 2014).

Antropometria é uma palavra grega (antropo que significa homem e; metria significa medida), pode ser definida como o estudo das medidas do homem, ou ainda como "o estudo das medidas físicas do corpo humano". Em vários períodos da história humana, podem-se constatar grandes obras realizadas por estudiosos e amantes da ciência e da anatomia, que tiveram como principal referência as medidas do corpo humano, como o "O Homem Vitruviano" de Leonardo Da Vinci. Ela estuda as medidas de tamanho e proporções do corpo humano. As medidas antropométricas tais como peso, altura, circunferência de cintura e circunferência de quadril são utilizadas para o diagnóstico do estado nutricional (desnutrição, excesso de peso e obesidade) e avaliação dos riscos para algumas doenças (diabetes mellitus, doenças do coração e hipertensão) em crianças, adultos, gestantes e idosos. (Manual de Antropometria, PNS, 2013). (Mussoi, 2014).

Outro fato importante das medidas corpóreas é ter como referência para projetos de produtos a levando em conta a postura do usuário. Os dados antropométricos definem as medições de tamanho, peso e proporções do corpo humano aplicáveis a um correto dimensionamento de projetos de produtos, equipamentos e postos de trabalho". Com o passar do tempo a necessidade de encontrar medidas mais exatas do ser humano foi crescendo cada vez mais. Hoje, para o desenvolvimento de produtos ergonômicos faz-se necessária a aplicação correta das dimensões humanas. Isso vem proporcionando uma crescente aplicação da Antropometria à Ergonomia. (Krause, 2005).

Em se tratando de exames laboratoriais, há uma crescente facilidade de acesso a esses devido à crescente incorporação de tecnologia aos serviços de saúde que estão expandindo o segmento da medicina diagnóstica. Tamanho crescimento traz consigo a velocidade de processamento dos dados e a maior facilidade de acesso a laboratórios, o que auxilia médicos exercerem os tratamentos devidos. Exames de sangue, glicemia, colesterol, urina, imagem, dentre outros, estão mais acessíveis e tornaram diagnósticos mais precisos e rápidos. Juntamente com os exames, crescem sofisticados sistemas de informação computadorizados para capturar, armazenar e distribuir os resultados. Há ainda a evolução nos diagnósticos mais precisos e com maior capacidade de prevenção de doenças, além de se ter maior tempo hábil para tratamento dos pacientes com doenças mais sérias. (Vasconcelos, 2008).

\section{Conclusão}

Desse modo, nota-se que a realização da avaliação nutricional da população infantil é primordial. A aferição do peso e altura permite a comparação de cada criança com o padrão de crescimento ideal para que se possa verificar a existência de possíveis deficiências. A avaliação antropométrica fornece dados importantes para o entendimento do estado geral da saúde de um paciente. Ela tem a vantagem de poder ser realizada em qualquer paciente, independentemente do estado de saúde e da idade. Além disso, o exame é simples, de baixo custo e pode ser feito no consultório. Além disso, quando associada ao ambiente escolar, a estratégia se torna ainda mais eficaz, haja vista a importância do trabalho multidisciplinar, de suma importância para evitar-se problemáticas nutricionais aos infantes. Assim, é importante que os trabalhos futuros, sob essa temática, se atentem que a prevenção deve ser a primeira linha de defesa contra as complicações decorridas de um estado nutricional inadequado.

\section{Referências}

Andrade, L. O. M., Barreto, I. C. C. C., Goya, N. \& Junior, T. M. (2004). A Estratégia Saúde da Família. Medicina ambulatorial: condutas de Atenção Primária baseadas em evidências. 3. ed. Porto Alegre: Artmed, 2004. cap. 07, 88-99.

Azevedo, A. L. M. \& Costa, A. M. (2010). A estreita porta de entrada do Sistema Único de Saúde (SUS): uma avaliação do acesso na Estratégia de Saúde da Família. Interface: Comunicação, Saúde, Educação, Botucatu/SP. 14 (35), 797-810. 
Research, Society and Development, v. 10, n. 9, e44210918351, 2021

(CC BY 4.0) | ISSN 2525-3409 | DOI: http://dx.doi.org/10.33448/rsd-v10i9.18351

Brasil. (2014). Ministério da Saúde, Secretaria de Atenção à Saúde, Departamento de Atenção Básica. Guia alimentar para a população brasileira. 2. ed. Brasília, DF: Ministério da Saúde.

Fonseca, V. M. (2014). Aspectos da situação nutricional brasileira. Revista Ciência e Saúde Coletiva. 19 (5), $1328-1329$.

Freitas, M. M. P., Pascoal, G. T. P., Santos, J. O., de Jesus, L. I., dos Santos, S. M., da Silva, V. A., Bastos, L. C. S., \& Matozinhos, F. P. (2018). Avaliação Nutricional Em Crianças De Uma Creche Em Minas Gerais: Relato De Experiência. Revista Contexto \&Amp; Saúde, 18 (34), 36-42.

Henriques, P., Sally, E. O., Burlandy, L. \& Beiler, R. M. (2012). Regulamentação da propaganda de alimentos infantis como estratégia para a promoção da saúde. Revista Ciência e Saúde Coletiva, 17 (2), 481-490.

Lima, B. R. (2010). A influência da mídia no comportamento infantil. Brasília: FATECS.

Mahan, L. K., Escott-Stump, S. \& Krause, S. (2005). Alimentos, Nutrição \& Dietoterapia. São Paulo, Roca.

Mello, E. D. (2002). O que significa avaliação do estado nutricional. Jornal de Pediatria. 75 (5), 357-358.

Moura, N. C. (2010). Influência da mídia no comportamento alimentar de crianças e adolescentes. Revista Segurança Alimentar e Nutricional, 17 (1), 113 122 .

Mussoi, T. D. (2014). Avaliação nutricional na prática clínica: da gestação ao envelhecimento. $1^{\text {a }}$ ed. Rio de Janeiro: Guanabara Koogan.

Porto, A. C. V., Pires, B. A. B. \& Coelho, S. C. (2013). Frequência de consumo de fast food em crianças de uma escola pública e uma escola privada do município de Nova Iguaçu no Rio de Janeiro e sua influência no perfil nutricional. Acta Pediatr Port, 44 (6), $301-305$.

Rodrigues, A. S., Carmo, I., Breda, J. \& Rito, A. I. (2011). Associação entre o marketing de produtos alimentares de elevada densidade energética e a obesidade infantil. Rev. Port Saúde Pública, 29 (2), 180-187.

Rodrigues, V. M. \& Fiates, G. M. R. (2012). Hábitos alimentares e comportamento de consumo infantil: influência da renda familiar e do hábito de assistir à televisão. Revista de Nutrição, 25 (3), 353-363.

Sampaio, L. R., Silva, M. C. M., Oliveira, T. M. \& Leite, V. R. (2012). Semiologia nutricional. EDUFBA, 23-47.

Saúde, Brasil. (1999). Nutrição pré-escolar \& escolar São Paulo: Aguilla, 28 min.

Starfield, B. (2002). Atenção Primária: equilíbrio entre necessidades de saúde, serviços e tecnologia. Ministério da Saúde. Brasil.

Vasconcelos, F. A. (2008). Avaliação nutricional de coletividades. Florianópolis: UFSC.

Vasconcelos, M. J. O. B., Barbosa, J. M., Pinto, I. C. S., Lima, T. M. \& Araújo, A. F. C. (2011). Nutrição clínica obstetrícia e pediatria. Rio de Janeiro: MedBook. 